\title{
性能数据驱动的机械产品关键设计参数识别方法“
}

\author{
褚学宁 陈汉斯 马红占 \\ (上海交通大学机械与动力工程学院 上海 200240)
}

\begin{abstract}
摘要: 利用运行数据识别产品设计缺陷或薄弱环节是产品开发和更新换代的主要模式, 也是保证产品服役性能稳定的重要途 径。提出了一种性能数据驱动的产品关键设计参数(薄弱环节) 识别方法。首先, 利用极限学习机算法划分运行工况, 提出了 基于核主成分分析和高斯混合模型的多工况性能退化评估方法, 消除了工况变化对性能退化评估的影响, 得到性能退化严重 的关键功能模块; 其次, 对性能监测数据进行聚类分析, 识别出与模块性能退化密切相关的关键性能监测参数; 最后, 建立 了 “性能监测参数一性能参数一设计参数” 三者之间的关联关系, 识别出导致性能严重退化的关键设计参数。以某大吨位履 带起重机作业机构为例，验证了方法的有效性。
\end{abstract}

关键词: 性能监测数据; 性能退化评估; 关键设计参数识别; 高斯混合模型; 履带起重机

中图分类号: TH122; TP391

\section{Identification of Critical Design Parameter for Mechanical Products Based on Performance Data}

\author{
CHU Xuening CHEN Hansi MA Hongzhan
}

(School of Mechanical Engineering, Shanghai Jiao Tong University, Shanghai 200240)

\begin{abstract}
Identifying the weaknesses or defects of the current design based on the operating data is the main mode of product development. It is also an important way to ensure the stable performance of products. An approach of critical design parameter identification based on performance data is proposed. Firstly, the operating conditions are identified by the algorithm of extreme learning machine. A performance degradation assessment method based on kernel principal component analysis and gaussian mixture model is conducted, which eliminates the influence of operating conditions on performance degradation assessment and obtains key functional modules with severe performance degradation. Secondly, cluster analysis is carried out on operation monitoring data to identify critical monitoring parameters closely related to module performance degradation. Finally, the correlation between "performance monitoring parameter - product performance parameter - design parameter" is established to identify the critical design parameters. The effectiveness of the proposed method is verified by a case study of a large tonnage crawler crane.
\end{abstract}

Key words: performance monitoring data; performance degradation assessment; critical design parameter identification; Gaussian mixture model; crawler crane

\section{0 前言}

随着科学技术的发展, 机械产品结构越来越复 杂, 运行工况环境也更加严酷和多变 ${ }^{[1]}$ 。经过一段 时间的运行, 产品性能可能会发生退化, 严重的情 况会导致设备故障或功能失效, 带来重大经济损 失 ${ }^{[2]}$ 。为了预防这一情况的发生, 通常采取对设备 进行状态监测(Condition monitoring, CM)的策略。

* 国家自然科学基金资助项目 $(51875345,51475290) 。 20200519$ 收到初 稿, 20200913 收到修改稿
即利用传感器采集设备运行过程中的性能数据, 分 析性能退化趋势, 预测剩余寿命, 实现预防性维护。 这类方法能有效地降低设备维护维修成本, 减少故 障发生概率。但是，更根本的措施，应该是在产品 设计阶段, 通过分析监测到的大量性能数据, 识别 导致性能退化的设计薄弱环节(即关键设计参数)并 加以改进, 来保证产品运行服役性能稳定可靠 ${ }^{[3-4]}$ 。

利用产品运行或使用过程中产生的数据来改进 和完善产品设计已有了一些研究。例如, 用户在使 用产品一段时间后会通过问卷、在线评论等方式发 表对产品的评论意见，厂商则通过对评论数据的分 
析、挖掘, 得到用户对产品的需求和改进建议, 将 其转化为新产品工程特性, 从而改进产品的功能或 性能 ${ }^{[5]}$ 。随着 “大数据科学” 的兴起以及传感器技 术的广泛应用, 以大范围、低成本、增量累积的方 式获取产品相关数据成为可能, 催生了基于用户产 品交互行为数据的交互式设计 ${ }^{[6]}$ 、移情设 ${ }^{\left[{ }^{[7]}\right.}{ }^{\text {和传达 }}$

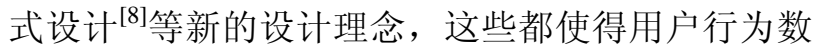
据对于改进产品设计具有了重要价值。但是, 这些 理念和方法大多是以提高用户满意度或解决产品缺 陷为目标的, 设计决策过程更多地关注用户主观评 价数据或行为数据, 且适用的对象大多是智能手机、 冰箱和汽车座椅等消费类产品。对于机械装备类产 品, 研究较多的是基于故障或失效数据来进行产品 设计改进。例如, 失效模式和影响分析(Failure mode and effects analysis, FMEA) ${ }^{[9]}$, 失效模式、影响和 危害性分析 (Failure mode effect and criticality analysis, FMECA) ${ }^{[10]}$ 和故障树分析 (Failure tree analysis, FTA $)^{[11]}$ 等方法, 主要是分析设备失效或发 生故障的原因来改进设计。然而, 随着技术成熟度 和可靠性的不断提高, 产品运行寿命大大延长, 能 采集到的故障或失效数据越来越少。与此相反, 产 品运行过程中状态性能数据越来越容易获得。经过 长期的积累这些数据基本涵盖了寿命期内各种可能 的工况环境, 真实地反映了产品运行过程中性能状 态变化的全过程, 蕴含了产品结构与性能之间的内 在关系, 数据量大、信息完整可靠。因此, 怎样在 少量故障数据基础上, 通过对性能数据的分析、挖 掘, 识别出导致性能严重退化或故障的设计薄弱环 节, 已成为亟待研究的课题。

据此, 提出了性能数据驱动的机械产品关键设 计参数识别方法。首先, 为了消除工况变化对性能 退化评估的影响, 提出了基于极限学习机算法的工 况识别方法。利用核主成分分析和高斯混合模型评 估各工况下产品性能退化情况, 得到性能退化严重 的关键功能模块。其次, 对性能监测数据进行聚类 分析, 识别出与模块性能退化密切相关的关键性能 监测参数。最后, 利用 “性能监测参数一性能参数 一设计参数” 三者之间的关联关系, 识别出导致性 能严重衰退的关键设计参数。

\section{1 多工况下性能退化评估}

产品运行过程中往往要经历多种工况, 不同工 况下性能退化程度是不同的。如果不加以区分, 采 用同一个阈值来判断性能退化严重程度可能会导致
误判或漏判情况发生。因此, 需要首先识别不同的 工况, 然后针对每个工况建立各自的性能退化评估 模型。在进行性能退化评估时, 对于新输入的性能 监测数据, 首先确定其所属的工况, 然后调用该工 况下的性能退化评估模型来评价性能的衰退程度。

\section{1 产品运行工况识别}

识别运行工况的目的是将产品整个运行周期采 集的性能监测数据划分为若干个子集，每个子集对 应于为一个典型工况。运行监测数据包含工况监测 数据和性能监测数据两类, 前者记录了产品的工作 环境和负载等信息, 可以作为运行工况识别的输入 数据。为了将各工况的数据分离并确定它们所属工 况, 采用基于极限学习机(Extreme learning machine, ELM)的方法。极限学习机是机器学习领域中的一种 人工神经网络模型, 是一种求解单隐层前馈神经网 络的快速学习算法。相比于传统的神经网络, 在保 证学习精度的前提下该算法拥有更快的学习速度, 可以较好地逼近工况监测数据和工况类型之间的非 线性映射关系(如图 1 所示)。



图 1 基于极限学习机算法的运行工况识别

神经网络输入层的神经元个数根据性能监测数 据样本的维度确定。假设有 $N$ 个监测时刻点, 每个 时刻点的样本记为 $\left(\boldsymbol{x}_{i}, \boldsymbol{y}_{i}\right)$, 其中 $\boldsymbol{x}_{i}$ 为 $n$ 维输入数据 矢量 $\boldsymbol{x}_{i}=\left(x_{i 1}, x_{i 2}, \cdots, x_{i n}\right)^{\mathrm{T}}, y_{i}$ 为对应的 $m$ 维输出结 果属性矢量 $\boldsymbol{y}_{i}=\left(y_{i 1}, y_{i 2}, \cdots, y_{i m}\right)^{\mathrm{T}}$, 其中 $i=1,2, \cdots, N$ 。 同时假设标准单隐层前馈神经网络的隐藏层神经元 个数为 $S$, 采用的激活函数记为 $\sigma(x)$, 那么标准单 隐层前馈神经网络的模型可由式(1)表示

$$
\sum_{i=1}^{S} \boldsymbol{\beta}_{i} \sigma_{i}\left(x_{j}\right)=\sum_{i=1}^{S} \boldsymbol{\beta}_{i} \sigma_{i}\left(w_{i} x_{j}+b_{i}\right)=o_{j}
$$

式中, $\boldsymbol{w}_{i}=\left(w_{i 1}, w_{i 2}, \cdots, w_{i n}\right)^{\mathrm{T}}$ 为输入样本与第 $i$ 个隐 层单元之间的权重, $b_{i}$ 为隐含层第 $i$ 个单元的偏移, $\boldsymbol{\beta}_{i}=\left(\beta_{i 1}, \beta_{i 2}, \cdots, \beta_{i m}\right)^{\mathrm{T}}$ 为第 $i$ 个隐层单元和输出结果 
之间的权重, $\boldsymbol{o}_{i}=\left(o_{i 1}, o_{i 2}, \cdots, o_{i m}\right)^{\mathrm{T}}$ 为标准单隐层前 馈神经网络的第 $j$ 个输出结果。含有 $S$ 个隐层神经 单元的标准单隐层前馈网络可以最小误差(可以为 零误差) 地逼近 $N$ 个监测点的采样数据, 即有 $\sum_{j=1}^{S}\left\|\boldsymbol{o}_{j}-\boldsymbol{y}_{j}\right\|=0$ 成立, 即存在网络参数矢量 $\boldsymbol{w}_{i}$, $b_{i}$ 和 $\boldsymbol{\beta}_{i}$ 使得下式成立

$$
\sum_{i=1}^{S} \boldsymbol{\beta}_{i} \sigma_{i}\left(\boldsymbol{w}_{i} \boldsymbol{x}_{j}+b_{i}\right)=\boldsymbol{y}_{j}
$$

训练和更新极限学习机的步骤如下:

步骤 1: 随机生成输入层与隐含层之间的权重 和隐含层神经元偏移。

步骤 2: 确定隐层神经元个数和激活函数, 计 算隐含层输出矩阵。

步骤 3: 计算输出层权重。

训练时, 输入的工况监测数据样本均为有标签 (已知工况)数据, 完成后可得到基准 ELM 模型。使 用时, 将待处理的工况监测数据输入模型中, 得到 的输出 $\boldsymbol{y}_{i}=\left(y_{1}, y_{2}, \cdots, y_{R}\right)^{\mathrm{T}}$ 即为工况识别结果。

\section{2 多工况下性能退化评估}

假设某个功能模块的性能状态可以由 $m$ 个性能 监测参数的测量值描述, 记采集到的原始性能监测 参数测量值数据集为 $\boldsymbol{x}_{m \times n}=\left(x_{1}, x_{2}, \cdots, x_{n}\right)$, 其中 $\boldsymbol{x}=\left(x_{1}, \cdots, x_{i}, \cdots x_{m}\right)$ 是一个 $m$ 维的数据矢量, $n$ 是 总的监测时刻点数量。 $p_{i}$ 是在某个特定监测时刻第 $i$ 个性能监测参数的测量值(其中, $i=1,2, \cdots, m, m$ 是 该功能模块性能监测参数的数量)。为了降低数据之 间的量纲差异和原始数据计算的误差, 采用 $z$-score 标准化公式对数据进行标准化处理, 如式(3)所示

$$
\bar{x}_{i}^{j}=\frac{x_{i}^{j}-\mu\left(\sum_{j=1}^{n} x_{i}^{j}\right)}{\sigma\left(\sum_{j=1}^{n} x_{i}^{j}\right)}
$$

式中, $x_{i}^{j}$ 为第 $i$ 个性能监测参数在第 $j$ 个性能监测 时刻的测量值(其中, $i=1,2, \cdots, m, j=1,2, \cdots, n$ ), $\mu\left(\sum_{i=1}^{n} x_{i}^{j}\right)$ 为第 $i$ 个性能监测参数所有监测时刻点数 据的平均值, $\sigma\left(\sum_{i=1}^{n} x_{i}^{j}\right)$ 为第 $i$ 个性能监测参数所有 监测时刻点数据的标准差, $\bar{x}_{i}^{j}$ 为数据标准化处理后 得到的数值, 经标准化处理后的时间序列为 $\boldsymbol{x}_{m \times n}^{\prime}=\left(x_{1}^{\prime}, x_{2}^{\prime}, \cdots, x_{n}^{\prime}\right)$ 。考虑到工况变动对性能退化分 析的影响, 提出基于混合高斯模型(Gaussian mixture model, GMM)的功能模块性能退化评估方法, 具体 流程如图 2 所示。

步骤 1：运行工况识别

采用 1.1 节所述基于 ELM 的工况识别方法识别 每个功能模块在不同性能监测时刻点所属的工况。

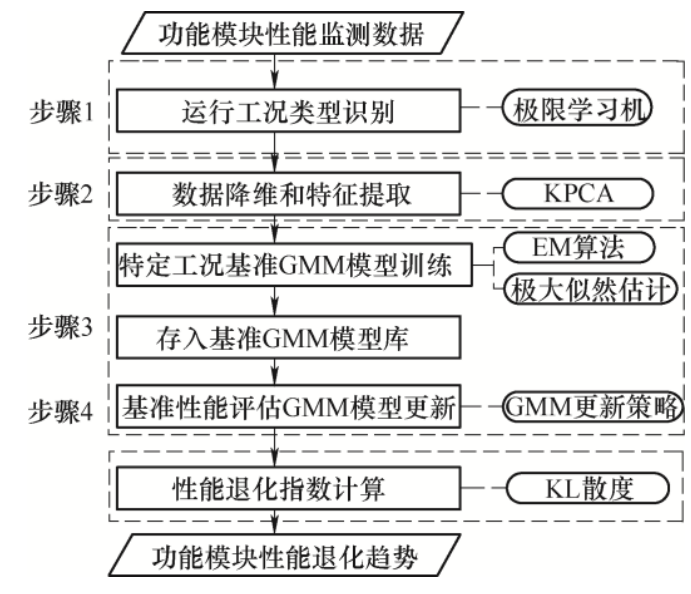

图 2 基于工况划分的性能退化评估

步骤 2: 性能监测数据特征提取

采用核主成分分析(Kernel principal component analysis, KPCA)方法 ${ }^{[13]}$ 提取性能监测数据集的敏感 数据特征, 将高维性能监测数据集转换为低维数据 集。首先，使用高斯径向基核函数(Gaussian radial basis function, GRBF)计算得到核矩阵 $\boldsymbol{K}$ 。然后，基 于核矩阵计算中心化核矩阵 $\boldsymbol{K}^{\mathrm{C}}=\boldsymbol{P} \boldsymbol{K} \boldsymbol{P}^{-1}$, 并将其分 解。其中, $\boldsymbol{P}=\boldsymbol{I}-\boldsymbol{e} \boldsymbol{e}^{\prime} / M$, 此处 $\boldsymbol{I}$ 是一个 $M \times M$ 维 的单元矩阵, $\boldsymbol{e}$ 是一个 $M$ 维的单元矢量。最后, 提 取性能特征矩阵的主元特征矢量, 生成新的特征数 据矩阵 $\boldsymbol{Y}=\left(\boldsymbol{y}_{1}, \cdots, \boldsymbol{y}_{i}, \cdots, \boldsymbol{y}_{m}\right)$, 将其作为训练和更新 GMM 模型和评估产品性能退化的输入数据。

步骤 3: GMM 模型构建和更新

在特定工况下, 进行功能模块健康状态 GMM 基准模型构建和模型更新。GMM 模型可以精确刻 画性能监测参数测量值的多态随机分布特点。GMM 模型通过对多个高斯单元模型的加权综合可以处理 多维度性能监测参数的复杂数据集, 适合用来描述 和评估运行工况复杂多变的履带起重机各功能模块 性能变动。混合高斯模型的概率密度函数为 ${ }^{[14]}$

$$
\begin{gathered}
\Phi(\boldsymbol{x})=\sum_{j=1}^{J} w_{i} \Phi_{j}(\boldsymbol{x})=\sum_{j=1}^{J} w_{i} \Phi_{j}\left(\boldsymbol{x} ; \boldsymbol{\mu}_{j}, \Sigma_{j}\right)= \\
\sum_{j=1}^{J} w_{i} \frac{1}{(2 \pi)^{Z / 2}\left|\Sigma_{j}\right|^{1 / 2}} e^{-\frac{1}{2}\left(\boldsymbol{x}-\boldsymbol{\mu}_{j}\right)^{\mathrm{T}}\left(\Sigma_{j}\right)^{-1}\left(\boldsymbol{x}-\boldsymbol{\mu}_{j}\right)}
\end{gathered}
$$

式中, $\Phi(x)$ 为一个面向时序性能监测参数的数据训 练集 $\left\{\boldsymbol{x}^{1}, \cdots, \boldsymbol{x}^{z}, \cdots, \boldsymbol{x}^{Z}\right\}$ 的概率密度函数, $Z$ 为性能监 测参数数据样本的数量, $\boldsymbol{x}$ 为某个监测时刻的 $m$ 维 特征数据矢量 $\left(x_{1}, x_{2}, \cdots, x_{m}\right)^{\mathrm{T}}$ 。在 GMM 模型的混合 概率密度函数中， $J$ 为高斯单元的数目， $w_{j}$ 为第 $j$ 个高斯单元的权重, $\boldsymbol{\mu}_{j}$ 为单个高斯密度函数的均值, $\Sigma_{j}$ 为单高斯模型之间的协方差矩阵。以上 GMM 模 
型中的参数均可通过经典的最大期望值算法 (Expectation maximum, EM) 求解得到 ${ }^{[15]}$ 。

在不同运行工况下, 建立不同的基准 GMM 模 型。将功能模块在各工况下性能正常状态训练数据 集 $\boldsymbol{X}^{(0)}$ 下训练得到的基准 GMM 模型记为 $\boldsymbol{\Omega}^{0}$, 相 应的模型参数记为 $\boldsymbol{\Omega}^{0}=\left(\Omega_{1}^{0}, \cdots, \Omega_{j}^{0}, \cdots, \Omega_{J}^{0}\right)$ 。如果 $\boldsymbol{X}^{(0)}=\left\{\boldsymbol{x}^{1}, \cdots, \boldsymbol{x}^{z}, \cdots, \boldsymbol{x}^{Z}\right\}$ 是性能监测参数数据特征 集的前 $Z$ 个数据特征样本 (即监测时刻点 $t_{1}$ 到 $t_{Z}$ 过程 中的特征数据集), 那么训练得到的 GMM 模型 $\boldsymbol{\Omega}^{0}$ 可以作为 $Z$ 个性能特征样本 $\left\{\boldsymbol{x}^{1}, \cdots, \boldsymbol{x}^{z}, \cdots, \boldsymbol{x}^{Z}\right\}$ 的基 准 GMM 模型。这里, $Z$ 可以根据具体的案例由设 计师决定。当监测时刻 $t_{Z+1}$ 采集到的新数据特征矢 量 $\boldsymbol{x}^{Z+1}$ 输入已有特征样本集时, 基准 GMM 模型 $\boldsymbol{\Omega}^{0}$ 需要及时做出参数更新和模型调整以反映能监测参 数特征数据集的变动。新的 $\mathrm{GMM}$ 模型应当在覆盖 特征数据矢量 $\boldsymbol{x}^{Z+1}$ 的基础上进行更新。因此, 采用 基于滑动时间窗的策略对 GMM 模型进行更新, 其 更新机制如下: 每当一个新监测时刻的数据特征矢 量 $\boldsymbol{x}^{Z+1}$ 作为最新数据矢量加入性能监测参数数据 特征矩阵 $\boldsymbol{X}^{(0)}=\left\{\boldsymbol{x}^{1}, \cdots, \boldsymbol{x}^{z}, \cdots, \boldsymbol{x}^{Z}\right\}$ 最后一列时, 就将 特征数据矩阵 $\boldsymbol{X}^{(0)}$ 中的第一个数据矢量 $\boldsymbol{x}^{1}$ 移除。同 时, 将新得到的特征数据集记为 $\boldsymbol{X}^{(1)}$, 此时用于训 练新 GMM 模型的特征数据集的性能特征数据矢量 数目仍为 $Z$ 。

步骤 4: 性能退化指数计算

通过计算和基准模型之间的距离, 可以得到该 功能模块性能水平在每个时刻点偏离正常状况的程 度。采用信息熵 KL 散度(Kullback-Leibler divergence) 方法 ${ }^{[16]}$ 计算不同监测时刻 GMM 模型之间的距离。 在特定工况下, 分别计算所有监测时刻的 GMM 模 型与该工况下的基准 GMM 模型的距离, 将这些距 离值按时间序列连接绘制, 可以得到各功能模块性 能衰退指数趋势变化曲线。通过对比不同功能模块 性能退化趋势, 可以识别出性能退化严重的功能模 块, 即关键功能模块。

\section{2 关键性能监测参数识别}

为了指导产品的设计改进, 除了确定性能退化 严重的关键功能模块, 还需要进一步识别对性能退 化敏感的性能监测参数, 以进一步找到与这些参数 相关的设计参数。通过对关键功能模块的所有性能 监测参数数据进行聚类分析, 识别出关键性能监测 参数, 包括功能故障时刻点计算和性能监测参数重 要度计算两个步骤。

\section{1 功能故障时刻点计算}

在某监测时刻, 如果一个功能模块的性能退化 指数值较大, 这意味着该功能模块的性能发生了较 大的变动, 将该时刻定义为功能故障时刻点。考虑 到性能退化的波动性，采用基于置信区间的自适应 阈值来定义功能故障时刻。第 $j$ 个功能模块性能退 化指数在高斯混合模型参数优化更新的时间窗口内 的均值 $\mu\left(d_{j}, k\right)$ 和方差 $\sigma^{2}\left(d_{j}, k\right)$ 分别根据式(5)和式 (6)计算得到

$$
\begin{gathered}
\mu\left(d_{j}, k\right)=\left.\frac{1}{n} \sum_{i=1}^{n} d_{j i}(k)\right|_{d_{j i}} \\
\sigma^{2}\left(d_{j}, k\right)=\left.\frac{1}{n-1} \sum_{i=1}^{n}\left[d_{j i}(k)-\mu\left(d_{j i}, k\right)\right]^{2}\right|_{d_{j i}}
\end{gathered}
$$

式中, $d_{j i}$ 为第 $j$ 个功能模块在 $t_{i}$ 时刻的性能退化指 数, $k$ 为混合高斯模型在参数优化和模型更新时间 窗的大小, 即每个时间窗内包含的监测时刻数。随 着时间窗向前移动, 性能退化指数的均值和方差会 同步动态变化, 从而使得性能退化指数的阈值能够 随着时间窗的移动自适应变化。性能退化指数均值 在 $(1-\alpha)$ 置信度下的置信区间可以由式(7)表示

$$
P\{\bar{\mu}-z \alpha<\mu<\bar{\mu}+z \alpha\}=1-\alpha
$$

式中， $\alpha$ 为置信水平， $z$ 为与置信区间相关的一致 性系数。在工程应用中, 置信度 $(1-\alpha)$ 通常选择为 95\% 99\%。在本研究中, 设置置信度为 96\%, 对应 的一致性系数 $z=2.06$ 。性能退化指数在时刻 $t_{i}$ 的上 下界阈值可由式(8)计算得到

$$
J_{t h}=\mu\left(d_{j i}, k\right) \pm 2.06 \sigma^{2}\left(d_{j i}, k\right)
$$

当 $d_{j i}$ 超出 $J_{t h}$ 的阈值范围时, 可以认为监测时 刻 $t_{i}$ 为该功能模块的功能故障时刻点。基于这个规 则, 可以找出所有性能退化指数超出阈值的时刻点, 形成功能故障时刻点的集合。

\section{2 性能监测参数重要度计算}

步骤 1: 选取某个关键功能模块, 提取与之相关 的原始性能监测数据。

步骤 2: 剔除关键功能模块功能故障时刻点的所 有性能监测参数数据样本点, 得到一个新的数据集。

步骤 3: 性能监测数据聚类分析。采用模糊 C均值方法 ${ }^{[17]}$ 对上一步得到的新数据集进行聚类。假 设经过聚类得到了 $R$ 个数据簇和数据中心, 则分别 记为 $\left\{D_{h 1}, \cdots, D_{h r}, \cdots, D_{h R}\right\}$ 。

步骤 4: 定义和计算与关键功能模块相关的性 能监测参数的正常取值范围。这里, 假设在第 $r$ 个 数据类中, 第 $h$ 个性能监测参数 $D_{h}$ 的正常取值范围 为 $\left[D_{h r}^{l}, D_{h r}^{u}\right], D_{h r}^{l}$ 是第 $r$ 个数据类中性能监测参数 $D_{h}$ 的最小值, $D_{h r}^{u}$ 是第 $r$ 个数据类中性能监测参数 
$D_{h}$ 的最大值, $\mu_{h r}$ 和 $\sigma_{h r}$ 分别为第 $h$ 个性能监测参 数在第 $r$ 个数据类中的均值和标准差。这里仍然设 置置信度为 $96 \%$, 对应的一致性系数 $z$ 为 2.06 , 性 能监测参数 $D_{h}$ 在第 $r$ 个数据类中的取值范围为 $\mu_{h r} \pm 2.06 \sigma_{h r}$ 。

步骤 5: 统计性能监测参数在功能故障时刻点超 出正常取值范围的次数。将每个功能故障时刻点的数 据与步骤 4 中获得的正常取值范围进行对比, 如果功 能故障时刻点的某个性能监测参数的值超出了正常取 值范围, 则累计一次该性能监测参数的超出次数。

步骤 6: 关键性能监测参数识别。根据每个性 能监测参数累计超出正常取值范围的次数, 采用 式(9)求解每个性能监测参数的重要度

$$
W D_{h}=\frac{n_{h}^{a}}{\sum_{h=1}^{H} n_{h}^{a}} \quad h=1,2, \cdots, H
$$

式中, $n_{h}^{a}$ 为第 $h$ 个性能监测参数的超出次数, $H$ 为 所有性能监测参数的总超出次数。将关键功能模块 的所有性能监测参数的重要度按照从大到小的顺序 排序, 重要度较大的(也就是对性能退化敏感)性能 监测参数被定义为关键性能监测参数。

\section{3 关键设计参数识别}

关键设计参数指的是设计不合理的产品几何结 构或材料参数, 是设计的薄弱环节, 它导致了产品 运行过程中性能的严重退化。因此, 找出与退化严 重的性能紧密相关的设计参数是识别设计薄弱环节 的关键。但在现实中, 有些性能参数往往无法直接 检测到, 只能通过若干个性能监测参数(即传感器采 集到的数据)来间接计算或表征。这样, 我们可以依 据性能监测参数与性能参数之间的关联关系, 由关 键监测参数找到退化严重的性能参数, 即关键性能 参数。此外, 产品性能是由设计参数决定的, 每个 性能参数与若干个设计参数相关。因此, 可以依据 性能参数与设计参数之间的关联关系, 由关键性能 参数找到关键设计参数。也就是建立如图 3 所示的 “性能监测参数一性能参数一设计参数”两级关联矩 阵, 通过两级转换完成从关键监测参数到关键设计 参数的映射, 实现关键设计参数的识别。

图 3a 描述了性能监测参数与性能参数之间的 关联关系。其中, $R_{q h}$ 为第 $q$ 个性能参数与第 $h$ 个 性能监测参数之间的关联关系强度, $r_{q k}$ 为第 $q$ 个性 能参数与第 $k$ 个性能参数之间的自相关关系强度, $w_{h}$ 为第 $h$ 个性能监测参数的权重, 上述各参数之间 的关联关系强度和自相关关系强度均由设计专家依
据经验给出。 $w_{q}$ 为第 $q$ 个性能参数的权重, 由式(10) 计算得到, $R_{q h}^{\prime}$ 为综合性能参数自相关关系强度 $r_{q k}$ 和性能参数与性能监测参数关联关系强度 $R_{q h}$ 之后 的修正关联强度值，可由式(11)计算得到

$$
\begin{gathered}
w_{q}=\sum_{h=1}^{H} w_{h} \cdot R_{q h}^{\prime} \\
R_{q h}^{\prime}=\frac{\sum_{k=1}^{Q} R_{q k} \cdot r_{q k}}{\sum_{q=1}^{Q} \Sigma_{k=1}^{Q} R_{q h} \cdot r_{q k}}
\end{gathered}
$$

图 $3 \mathrm{~b}$ 描述了性能参数与设计参数之间的关联 关系。其中, $R_{q, l g}$ 为第 $q$ 个性能参数和第 $l$ 个关键 功能模块的第 $g$ 个设计参数之间的关联强度关系, $r_{l g, s v}$ 为设计参数之间的自相关关系强度。上述各参 数之间的关联关系强度和自相关关系强度均由设计 专家依据经验给出。 $w_{l g}$ 为第 $l$ 个关键功能模块的第 $g$ 个设计参数的重要度, 可通过式(12)得到。其中, $R_{q, l g}^{\prime}$ 为综合考虑了设计参数之间的自相关关系强度 $r_{l g, s v}$ 和设计参数与性能监测参数之间的关联关系强 度 $R_{q, l g}$ 后得到的修正关联关系强度, 可由式(13)计 算得到

$$
\begin{gathered}
w_{l g}=\sum_{q=1}^{Q} w_{q} \cdot R_{q, l g}^{\prime} \\
R_{q, l g}^{\prime}=\frac{\sum_{l=1}^{L} \Sigma_{l v}^{l G} R_{q, l v} \cdot r_{l g, s v}}{\sum_{q=1}^{Q} \Sigma_{l=1}^{L} \Sigma_{l v}^{l G} R_{q, l g} \cdot r_{l g, s v}}
\end{gathered}
$$

通过对比各个设计参数的重要度, 就可以识别 出与功能模块性能退化密切相关的关键设计参数。

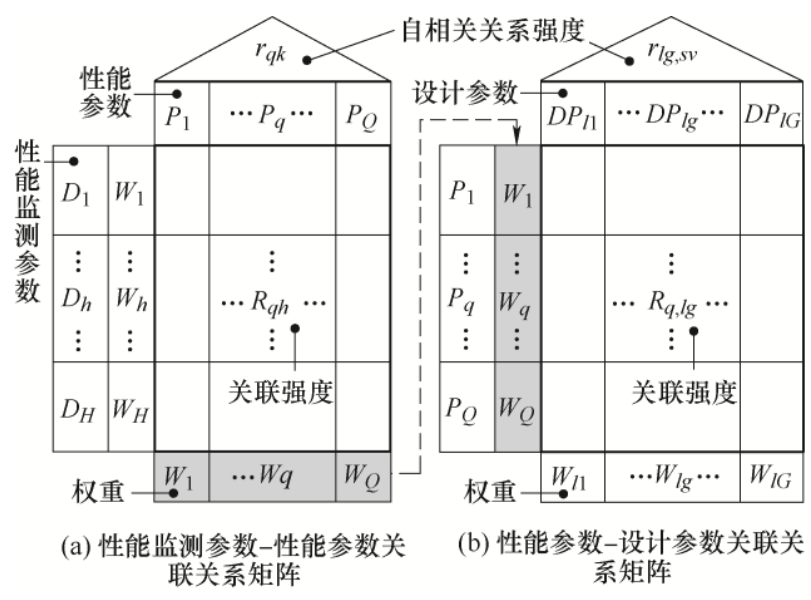

图 3 “性能监测参数一性能参数一设计参数” 两级 关联关系矩阵

\section{4 案例分析}

\section{1 案例研究}

选取某企业 SCC6300 型大吨位履带起重机作 为改进设计的案例。该型号履带起重机在运行过程 
中通过内置传感器采集了大量性能监测数据, 以表 格、文档和数据记录板等形式存于企业的设计和运 维部门。SCC6300 型履带起重机的主体作业机构如 图 4 所示, 运行时包括标准主臂工况(heavy main boom, SH)、轻型主臂工况(light main boom, LH)、 固定副臂工况(heavy main boom \& light fixed fly jib, SH \& LF)、塔式工况(heavy main boom \& luffing fly jib, SW)和超起工况(heavy main boom \& super lift, SSL)5 种典型工况。

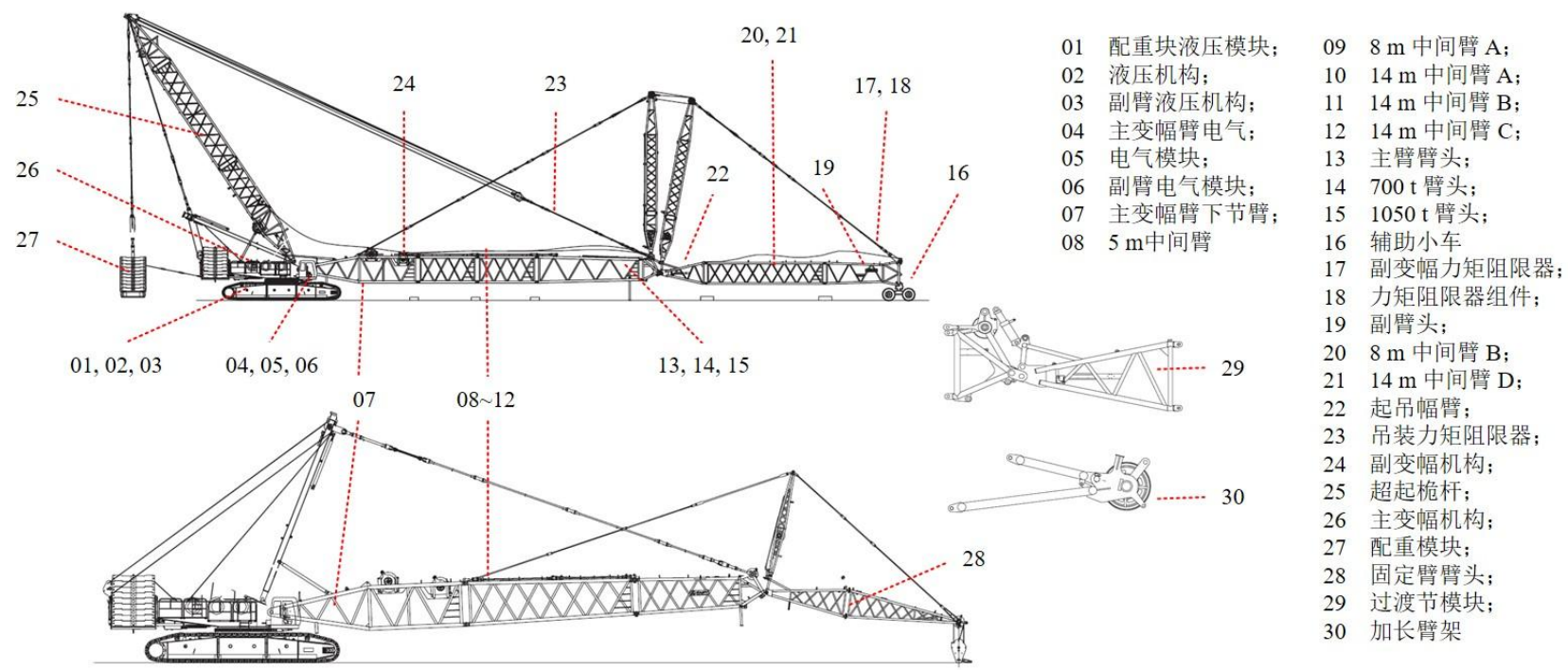

图 4 SCC6300 型履带起重机主体作业机构示意图

图 5 为由 30 个零部件构成的作业机构功能层次 结构, 功能模块之间包含信息流、能量流和物料流 等三种关联关系。这些关系将用于构建性能参数和 设计参数的关联关系矩阵模型。作业机构包含的功 能模块及性能监测参数如表 1 所示。性能监测数据 的采集时间区间为 2014 年 12 月 14 日 12:00:00 至
2015 年 3 月 9 日 24:00:00。数据采样频率是 10 分钟/ 次, 共计采集 6120 组有效数据样本。数据采集开 始时, 该型号履带起重机自出厂投入使用开始已经 平稳运行 10 天, 数据采集结束时, 产品部分功能结 构已经出现明显性能下降(中间运行过程中主要功 能未发生产品停机、停转和其他突发性功能故障)。

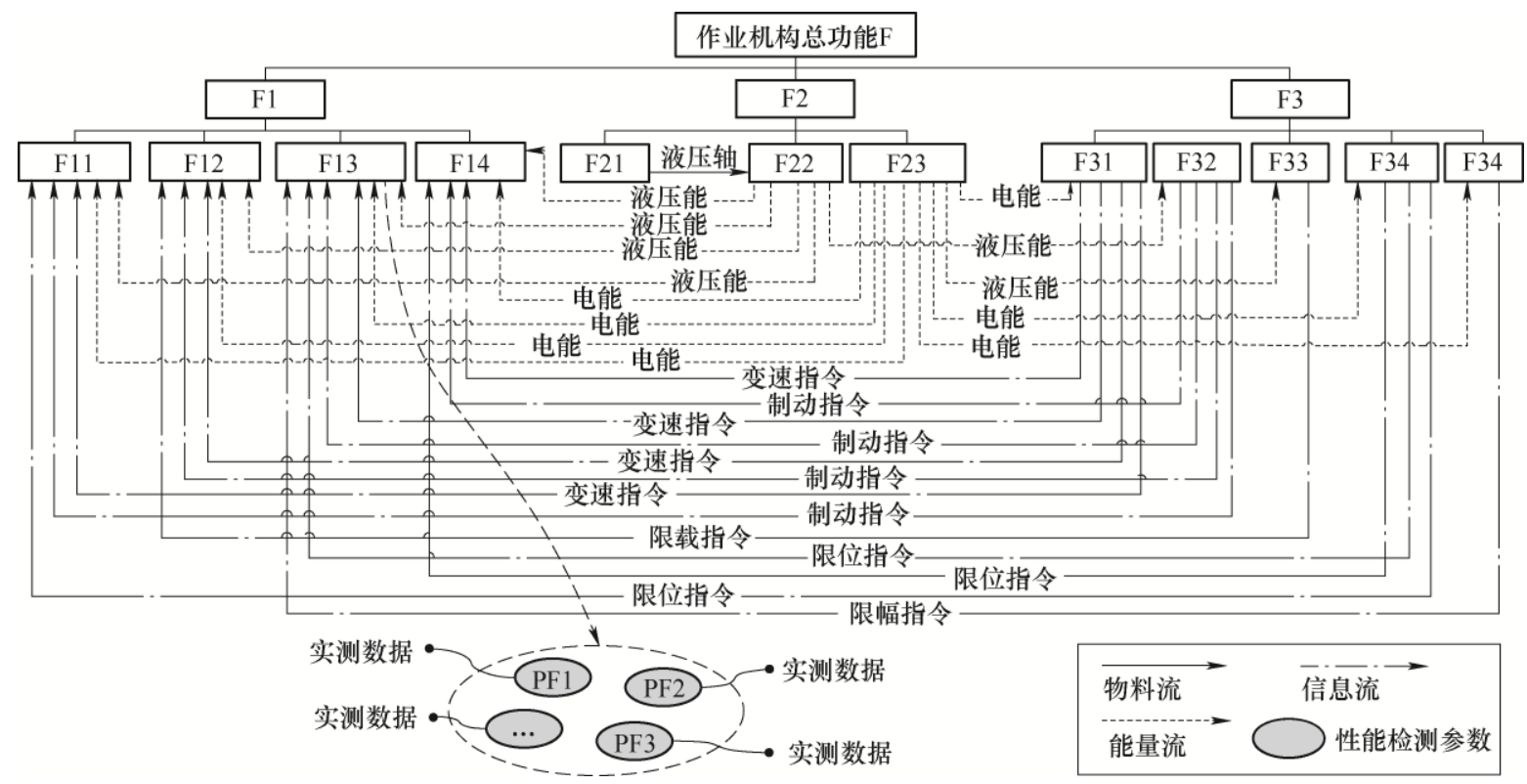

图 5 履带起重机作业机构的层次化功能结构模型 
表 1 SCC6300 履带起重机作业机构功能模块及性能监测参数

\begin{tabular}{|c|c|c|c|c|}
\hline $\begin{array}{l}\text { 一级功 } \\
\text { 能模块 }\end{array}$ & 功能描述 & $\begin{array}{l}\text { 二级功 } \\
\text { 能模块 }\end{array}$ & 功能描述 & 性能监测参数 \\
\hline \multirow{4}{*}{$\mathrm{F} 1$} & \multirow{4}{*}{$\begin{array}{l}\text { 运动执行 } \\
\text { 模块 }\end{array}$} & F11 & 回转运动 & $\begin{array}{l}\text { 回转速度 }\left(\mathrm{PF}_{1}\right) \text { 、回转轴力矩 }\left(\mathrm{PF}_{2}\right) \text { 、回转液压油回流量 }\left(\mathrm{PF}_{3}\right) \text { 、回转轴承温度 }\left(\mathrm{PF}_{4}\right) \text { 、回转主轴 } \\
\text { 温度 }\left(\mathrm{PF}_{5}\right) 、 \text { 回转轴承油液温度 }\left(\mathrm{PF}_{6}\right) \text { 、回转循环时长 }\left(\mathrm{PF}_{7}\right) \text { 、回转驱动力 }\left(\mathrm{PF}_{8}\right) \\
\end{array}$ \\
\hline & & F12 & 提升运动 & $\begin{array}{l}\text { 主臂提升速度 }\left(\mathrm{PF}_{1}\right) \text { 、驱动主轴温度 }\left(\mathrm{PF}_{2}\right) \text { 、提升力矩 }\left(\mathrm{PF}_{3}\right) \text { 、主支撑轴承轴温 }\left(\mathrm{PF}_{4}\right) \text { 、提升液压 } \\
\text { 油回流量 }\left(\mathrm{PF}_{5}\right) \text { 、主勾升速 }\left(\mathrm{PF}_{6}\right) \text { 起升葫芦升速 }\left(\mathrm{PF}_{7}\right)\end{array}$ \\
\hline & & F13 & 变幅运动 & 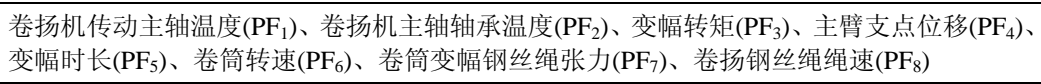 \\
\hline & & F14 & 伸缩运动 & $\begin{array}{l}\text { 伸缩装置液压油回流量 }\left(\mathrm{PF}_{1}\right) \text { 、伸缩液压恶排量 }\left(\mathrm{PF}_{2}\right) \text { 、伸缩机构主轴温度 }\left(\mathrm{PF}_{3}\right) \text { 、润滑液温度 } \\
\left(\mathrm{PF}_{4}\right) \text { 、伸缩液压油油压 }\left(\mathrm{PF}_{5}\right) \text { 、伸缩驱动力矩 }\left(\mathrm{PF}_{6}\right) \text { 、吊臂角 }\left(\mathrm{PF}_{7}\right) \text { 、副臂倾角 }\left(\mathrm{PF}_{8}\right)\end{array}$ \\
\hline \multirow{3}{*}{ F2 } & \multirow{3}{*}{$\begin{array}{l}\text { 能量供应 } \\
\text { 模块 }\end{array}$} & $\mathrm{F} 21$ & 机械-液压转换 & $\begin{array}{l}\text { 液压百转速 }\left(\mathrm{PF}_{1}\right) \text { 、液压百主轴温度 }\left(\mathrm{PF}_{2}\right) \text { 、液压泉主轴轴承温度 }\left(\mathrm{PF}_{3}\right) \text { 、液压流量 }\left(\mathrm{PF}_{4}\right) \text { 、马达 } \\
\text { 有功功率 }\left(\mathrm{PF}_{5}\right) \text { 、液压压力 }\left(\mathrm{PF}_{6}\right)\end{array}$ \\
\hline & & F22 & 液压供应 & $\begin{array}{l}\text { 液压油回油量 }\left(\mathrm{PF}_{1}\right) \text { 、液压马达转速 }\left(\mathrm{PF}_{2}\right) \text { 、马达有功功率 }\left(\mathrm{PF}_{3}\right) \text { 、液压压力 }\left(\mathrm{PF}_{4}\right) \text { 、液压阀启闭 } \\
\text { 力矩 }\left(\mathrm{PF}_{5}\right) \text { 、启闭速度 }\left(\mathrm{PF}_{6}\right) \text { 、液压排泄压力 }\left(\mathrm{PF}_{7}\right)\end{array}$ \\
\hline & & F23 & 电能供应 & $\begin{array}{l}\text { 输出电流 }\left(\mathrm{PF}_{1}\right) \text { 、输出电压 }\left(\mathrm{PF}_{2}\right) \text { 、输出电流频率 }\left(\mathrm{PF}_{3}\right) \text { 、电机转矩 }\left(\mathrm{PF}_{4}\right) \text { 、电动机主轴转速 }\left(\mathrm{PF}_{5}\right) \text { 、 } \\
\text { 电机主轴温度 }\left(\mathrm{PF}_{6}\right)\end{array}$ \\
\hline \multirow{5}{*}{ F3 } & \multirow{5}{*}{$\begin{array}{l}\text { 运动控制 } \\
\text { 模块 }\end{array}$} & F31 & 变速传动控制 & $\begin{array}{l}\text { 变速器输入轴轴温 }\left(\mathrm{PF}_{1}\right) \text { 、输出轴轴温 }\left(\mathrm{PF}_{2}\right) \text { 、连接轴轴温 }\left(\mathrm{PF}_{3}\right) 、 \text { 齿轮箱油液温度 }\left(\mathrm{PF}_{4}\right) \text { 、液压 } \\
\text { 驱动力 }\left(\mathrm{PF}_{5}\right) \text { 、传动扭矩 }\left(\mathrm{PF}_{6}\right)\end{array}$ \\
\hline & & F32 & 操作制动 & 制动距离 $\left(\mathrm{PF}_{1}\right)$ 、制动时间 $\left(\mathrm{PF}_{2}\right)$ 、制动减速度 $\left(\mathrm{PF}_{3}\right)$ 、制动力矩 $\left(\mathrm{PF}_{4}\right)$ \\
\hline & & F33 & 载荷限制 & 处理器温度 $\left(\mathrm{PF}_{1}\right)$ 、传感器温度 $\left(\mathrm{PF}_{2}\right)$ 、电容水平测量值 $\left(\mathrm{PF}_{3}\right)$ 、超限时间 $\left(\mathrm{PF}_{4}\right)$ \\
\hline & & F34 & 位移限制 & $\begin{array}{l}\text { 处理器温度 }\left(\mathrm{PF}_{1}\right) \text { 、传感器温度 }\left(\mathrm{PF}_{2}\right) \text { 、电容水平测量值 }\left(\mathrm{PF}_{3}\right) \text { 、限位臂转角 }\left(\mathrm{PF}_{4}\right) \text { 、限位器操作 } \\
\text { 力矩 }\left(\mathrm{PF}_{5}\right)\end{array}$ \\
\hline & & F35 & 变幅限制 & 处理器温度 $\left(\mathrm{PF}_{1}\right)$ 、传感器温度 $\left(\mathrm{PF}_{2}\right)$ 、电容水平测量值 $\left(\mathrm{PF}_{3}\right)$ 、超限时长 $\left(\mathrm{PF}_{4}\right)$ \\
\hline
\end{tabular}

分别选取 5 种典型工况下的 100 组数据, 共计 500 组已知所属工况的数据用于构建工况识别模型。 其中 400 组数据用于训练极限学习机模型, 剩余 100 组数据用于验证模型准确率。全连接层中输入层神 经元个数设置为 8 , 对应 8 个工况特征参数; 输出 神经元个数设置为 5 , 对应 5 个不同的工况类型; 隐含层神经元个数设置为 12 。使用 AdaGrad 算法作 为优化算法, 学习率设置为 0.001 , 迭代次数设置为 2 000。使用 Python 3.6 语言编写程序进行训练和测 试, 最终得到基于 ELM 的工况识别模型的输入层 与隐含层权重和偏移量参数(表 2), 隐含层与输出层 之间的权重和偏移量参数(表 3 )。

将 6120 组工况监测数据按照时间顺序, 逐一 输入到训练好的 ELM 模型中。ELM 模型可以识别 每个监测时刻起重机作业机构所处的工况状态, 如 图 6 所示。在划分工况时段以后, 需要对作业机构 的各功能模块进行多工况下的性能衰退评估。在输 入新监测时刻的性能监测参数数据输入时, 先确定 该时刻数据对应的工况, 并调用该工况下的基准高 斯混合模型训练该时刻对应的高斯混合模型; 最后, 计算每个时刻的高斯混合模型与对应工况下的基准 高斯混合模型之间的模型距离 KL 散度, 以得到各 个监测时刻的性能退化指数, 从而量化评估作业机 构各功能模块的性能退化程度。在构建每个工况的 基准 GMM 模型 $\mathrm{G}(0)$ 时, 采用最大期望值 EM 算法
和极大似然估计法确定基准 GMM 模型的模型参数 $\Omega(0)$ 。训练 GMM 模型时, 基准 GMM 模型的高斯 单元数量设置为 $6, \mathrm{EM}$ 算法在求解模型参数时循环 次数设置为 1000 。构建的 5 个典型工况的基准 GMM 模型可视化投影如图 7a 所示。

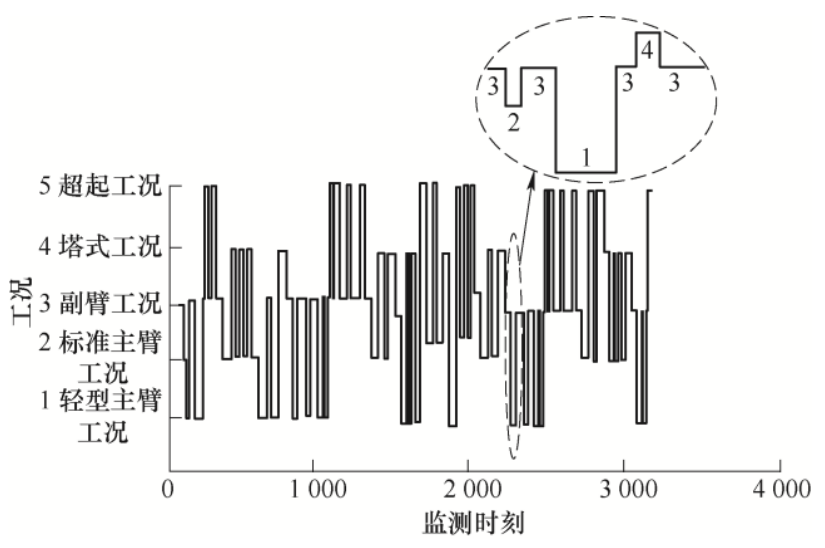

图 6 履带起重机作业机构运行工况识别结果

将降维后的时序性能数据逐个输入, 每个监测 时刻都会先识别其所属的运行工况，然后调用该工 况下最新一次生成的 GMM 模型对其进行更新, 以 描述新的性能监测时刻的数据分布情况。重复以上 过程, 可以得到功能模块 F13 在所有性能监测时刻 对应的 GMM 模型。由于篇幅限制, 在图 7b 中仅展 示了功能模块 F13 在副臂工况下不同时刻的 GMM 模型变动情况。不同工况下 GMM 模型的变动是由 
于 F13 功能模块的性能衰退造成的。随着各工况下 模块不同时刻的性能退化指数, 得到的性能退化曲 GMM 模型的更新变动, 采用 KL 散度计算各个功能线如图 8 所示。

表 2 工况识别模型的输入层与隐含层之间的权重和偏置参数

\begin{tabular}{|c|c|c|c|c|c|c|c|c|c|}
\hline & $\mathrm{CP} 1$ & $\mathrm{CP} 2$ & $\mathrm{CP} 3$ & $\mathrm{CP} 4$ & CP5 & CP6 & $\mathrm{CP} 7$ & CP8 & 偏置 $b_{1}$ \\
\hline H1 & -1.394 & -0.727 & -0.573 & -1.081 & -1.306 & -0.667 & -0.482 & -0.989 & -0.897 \\
\hline $\mathrm{H} 2$ & -1.273 & -1.307 & -1.032 & -1.310 & -1.201 & -1.170 & -0.974 & -1.217 & -0.714 \\
\hline $\mathrm{H} 3$ & -1.191 & -1.008 & -1.015 & -1.001 & -1.121 & -0.937 & -0.985 & -0.910 & -0.748 \\
\hline $\mathrm{H} 4$ & -1.189 & -0.683 & -0.756 & -1.310 & -0.979 & -0.684 & -0.772 & -1.298 & -0.838 \\
\hline H5 & -1.182 & -1.400 & -0.465 & -0.880 & -1.201 & -1.430 & -0.560 & -0.885 & -0.933 \\
\hline H6 & -1.179 & -0.189 & -1.398 & -0.510 & -0.828 & -0.022 & -1.356 & -0.417 & -0.859 \\
\hline $\mathrm{H} 7$ & -1.156 & -0.887 & -1.050 & -1.321 & -1.251 & -1.093 & -1.084 & -1.342 & -0.814 \\
\hline H8 & -1.125 & -0.644 & -0.861 & -0.797 & -1.312 & -0.632 & -0.869 & -0.342 & -1.075 \\
\hline H9 & -1.226 & -1.173 & --1.094 & -0.919 & -1.153 & -0.801 & -1.152 & -0.410 & -0.798 \\
\hline H10 & -1.160 & -1.227 & -0.947 & -0.657 & -1.412 & -0.127 & -0.850 & -0.680 & -0.671 \\
\hline H11 & -1.048 & -0.914 & -0.918 & -0.730 & -1.252 & -1.281 & -1.076 & -0.855 & -0.887 \\
\hline $\mathrm{H} 12$ & -0.908 & -1.178 & -0.857 & -1.122 & -1.060 & -0.641 & -1.237 & -0.871 & -0.931 \\
\hline
\end{tabular}

表 3 工况识别模型的隐含层与输出层之间的权重和偏置参数

\begin{tabular}{|c|c|c|c|c|c|c|}
\hline & WC1 & WC2 & WC3 & WC4 & WC5 & 偏置 $b_{2}$ \\
\hline $\mathrm{H} 1$ & -0.700 & -0.417 & 0.210 & -0.735 & -0.725 & 0.190 \\
\hline $\mathrm{H} 2$ & -0.378 & 0.096 & -0.400 & -0.802 & -0.228 & -0.284 \\
\hline $\mathrm{H} 3$ & -0.761 & -0.264 & -0.596 & -0.293 & 0.459 & 0.221 \\
\hline $\mathrm{H} 4$ & -1.006 & -0.024 & -0.070 & 0.061 & -0.057 & 0.120 \\
\hline H5 & -0.567 & 0.093 & 0.637 & 0.107 & -0.079 & 0.090 \\
\hline H6 & -0.177 & 0.481 & -0.264 & -0.592 & -0.005 & -0.284 \\
\hline $\mathrm{H} 7$ & -1.170 & 0.140 & 0.753 & -0.248 & -0.640 & 0.221 \\
\hline $\mathrm{H} 8$ & -0.059 & -0.090 & -0.541 & 0.479 & 0.626 & 0.120 \\
\hline H9 & -0.480 & -0.279 & 0.123 & -0.786 & -0.592 & 0.261 \\
\hline H10 & -0.118 & 0.246 & -0.712 & -0.847 & -0.486 & -0.170 \\
\hline H11 & -0.548 & -0.102 & -0.886 & -0.316 & -0.493 & 0.132 \\
\hline H12 & -0.882 & 0.109 & -0.246 & 0.023 & -0.381 & 0.378 \\
\hline
\end{tabular}

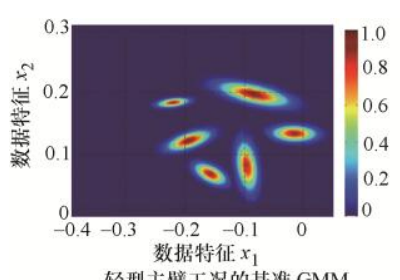

轻型主臂工况的基准 GMM

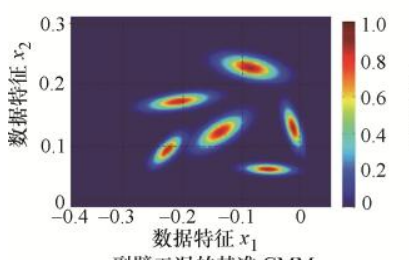

副臂工况的基准 $\mathrm{GMM}$

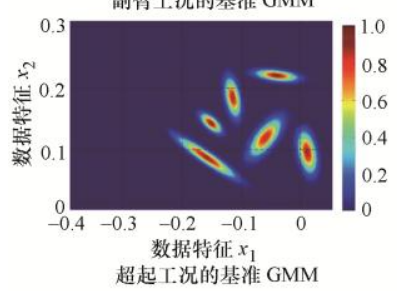

(a) 各工况的基准 GMM 模型

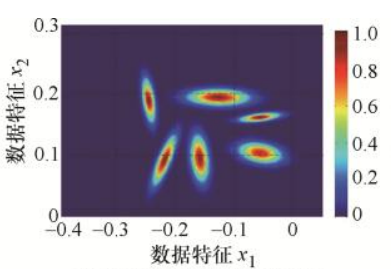

标准主臂工况的基准 GMM

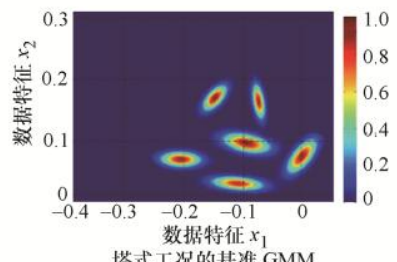

塔式工况的基准 $\mathrm{GMM}$

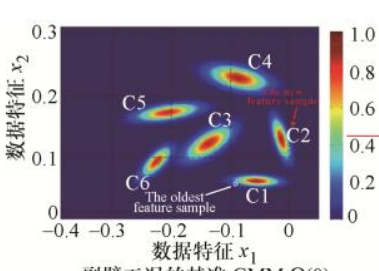

副臂工况的基准 GMM $\Omega(0)$

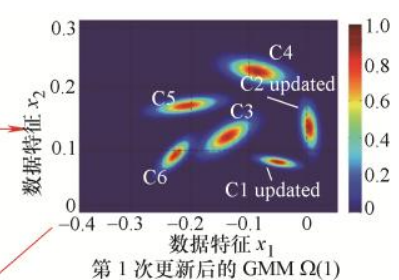

第 1 次更新后的 GMM $\Omega(1)$

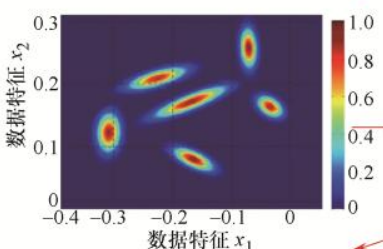

第 1000 次更据特后的 $x_{1}$ GMM $\Omega(1000)$

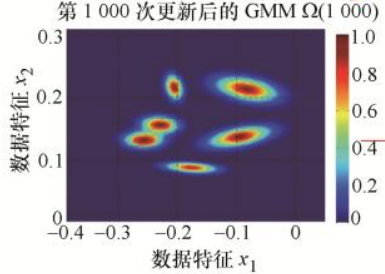

第 3000 次更新后的 GMM $\Omega(3000)$
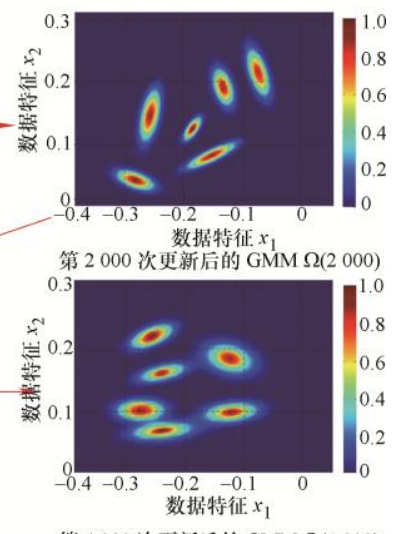

(b) 副臂工况下的 GMM 模型变动

图 7 'F13'模块的基准 GMM 模型和副臂工况下的 GMM 模型变动情况 


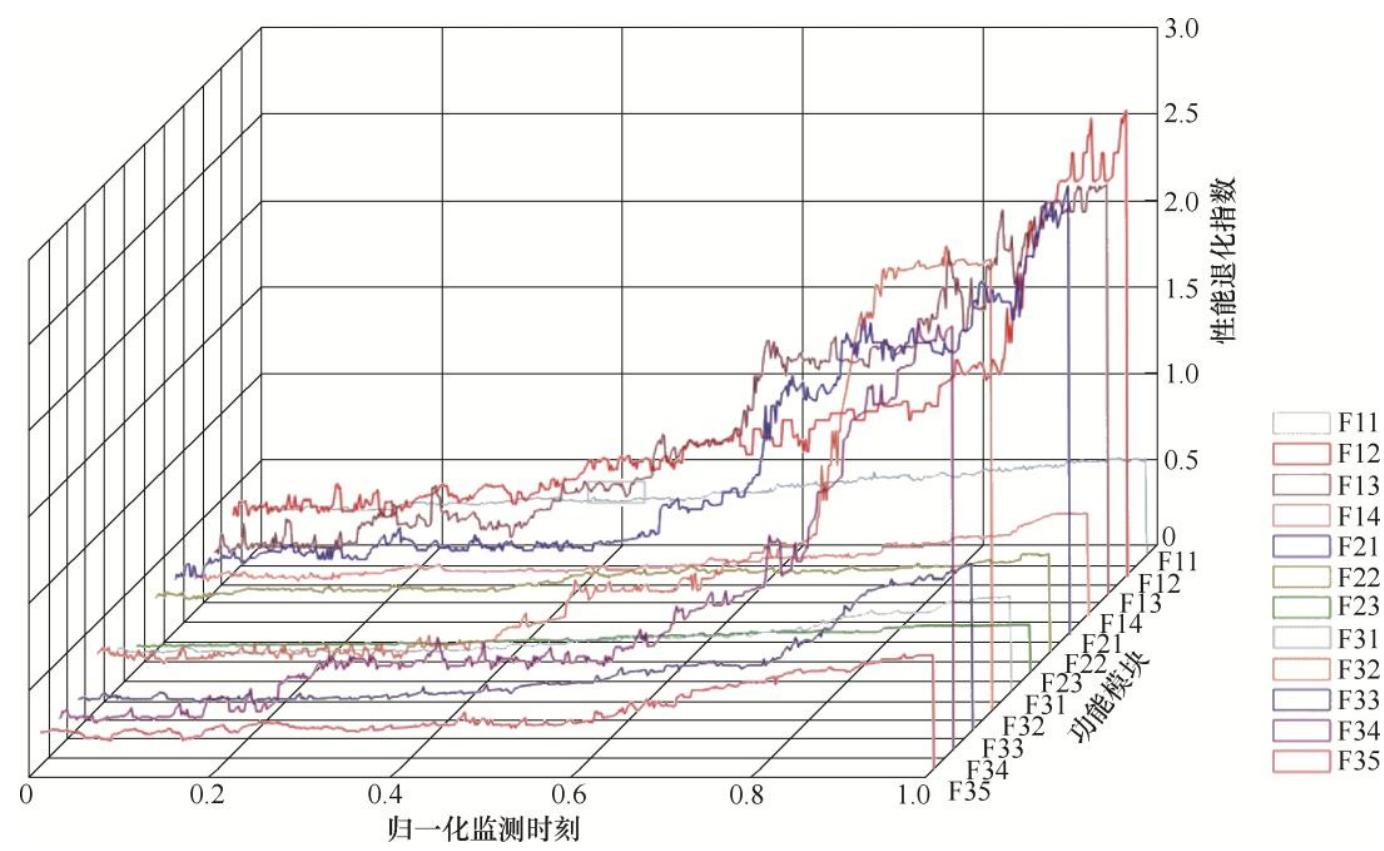

图 8 作业机构各功能模块的性能退化趋势曲线

以功能模块 $\mathrm{F} 13$ 为例, 根据性能监测数据的聚 类结果, 分别统计每个性能监测参数在功能故障时 刻点超出参考取值范围的次数, 并依此计算其归一 化后的重要度。计算结果见表 4 。根据重要度排序, 确定卷筒转速 $\left(\mathrm{PF}_{6}\right)$ 、卷筒变幅钢丝绳拉力 $\left(\mathrm{PF}_{7}\right)$ 、卷 扬钢丝绳绳速 $\left(\mathrm{PF}_{8}\right)$ 、起吊重量 $\left(\mathrm{C}_{1}\right)$ 和作业半径 $\left(\mathrm{C}_{4}\right)$ 为关键性能监测参数。

表 4 功能模块 F13 性能监测参数重要度

\begin{tabular}{lcc}
\hline \multicolumn{1}{c}{ 性能监测参数 } & 超出取值范围次数 & 归一化重要度 \\
\hline 传动主轴温度 $\mathrm{PF}_{1} /{ }^{\circ} \mathrm{C}$ & 96 & 0.0372 \\
主轴轴承温度 $\mathrm{PF}_{2} /{ }^{\circ} \mathrm{C}$ & 67 & 0.0260 \\
变幅转矩 $\mathrm{PF}_{3} /(\mathrm{kN} \cdot \mathrm{m})$ & 116 & 0.0450 \\
主臂支点位移 $\mathrm{PF}_{4} / \mathrm{cm}$ & 75 & 0.0291 \\
变幅时长 $\mathrm{PF}_{5} / \mathrm{min}$ & 41 & 0.0159 \\
卷筒转速 $\mathrm{PF}_{6} /(\mathrm{r} / \mathrm{min})$ & 391 & $\mathbf{0 . 1 5 1 ~ 6}$ \\
变幅钢丝绳张力 $\mathrm{PF}_{7} / \mathrm{kN}$ & 327 & $\mathbf{0 . 1 2 6 7}$ \\
卷扬钢丝绳绳速 $\mathrm{PF}_{8} /(\mathrm{m} / \mathrm{s})$ & 285 & $\mathbf{0 . 1 1 0 5}$ \\
起吊重量 $\mathrm{C}_{1} / \mathrm{t}$ & 359 & $\mathbf{0 . 1 3 9 1}$ \\
起吊物体体积 $\mathrm{C}_{2} / \mathrm{m}^{3}$ & 102 & 0.0395 \\
起升高度 $\mathrm{C}_{3} / \mathrm{m}$ & 83 & 0.0322 \\
作业半径 $\mathrm{C}_{4} / \mathrm{m}$ & 334 & $\mathbf{0 . 1 2 9 5}$ \\
风速 $\mathrm{C}_{5} / \mathrm{m} / \mathrm{s}$ & 94 & 0.0364 \\
风压 $\mathrm{C}_{6} / \mathrm{kPa}$ & 68 & 0.0264 \\
环境温度 $\mathrm{C}_{7} /{ }^{\circ} \mathrm{C}$ & 123 & 0.0477 \\
环境湿度 $\mathrm{C}_{8} /(\% \mathrm{Rh})$ & 19 & 0.0074 \\
\hline
\end{tabular}

作业机构变幅运动功能模块是多个产品性能的 载体, 结合设计工程师的经验, 给出了 F13 变幅运 动模块的 5 个产品性能要求, 分别为 “变幅平衡性 $\left(\mathrm{P}_{1}\right)$ ”、“变幅平滑性 $\left(\mathrm{P}_{2}\right)$ ”、“起幅抗冲击性 $\left(\mathrm{P}_{3}\right)$ ”、“落 幅操控性 $\left(\mathrm{P}_{4}\right)$ ” 和 “结构耐久性 $\left(\mathrm{P}_{5}\right)$ ”。该模块的 8 个主要设计参数分别为 “卷扬绳槽间距 $\left(\mathrm{DP}_{13,1}\right)$ ”、 “绳槽数量 $\left(\mathrm{DP}_{13,2}\right)$ ”、“出绳方式 $\left(\mathrm{DP}_{13,3}\right)$ ”、“筒径大 小 $\left(\mathrm{DP}_{13,4}\right)$ ”、“卷筒材质 $\left(\mathrm{DP}_{13,5}\right)$ ”、“卷扬钢丝绳类型 $\left(\mathrm{DP}_{13,6}\right)$ ”、“卷扬钢丝绳直径 $\left(\mathrm{DP}_{13,7}\right)$ ” 和 “钢丝绳材 质 $\left(\mathrm{DP}_{13,8}\right)$ ”。得到关键性能监测参数后, 首先建立 性能监测参数与性能参数之间的关联关系矩阵。根 据式(10)和(11), 结合性能监测参数重要度和设计师 给出的关联关系强度, 计算得到性能参数的权重。 然后建立性能参数与设计参数之间的关联关系矩阵, 根据式(12)和(13), 结合性能参数的权重和设计师给 出的关联关系强度, 计算得到设计参数的重要度。 最终得到的“性能监测参数一性能参数一设计参数” 两级关联关系矩阵如图 9 所示。设计参数重要度的 计算结果如图 9 最后一行所示, 据此对功能模块 F13 的设计参数进行排序。可以看出, 设计参数 “筒径 大小”、“绳槽数量” 和 “卷扬钢丝绳直径” 有较高 的重要度, 识别为关键设计参数。调整优化这些设 计参数是提高起重机作业机构的性能稳定性的关键。 


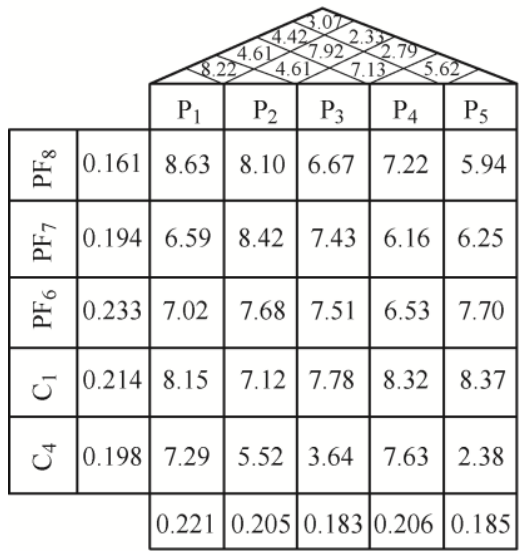

(a) 性能监测参数-性能参数关联关系矩阵

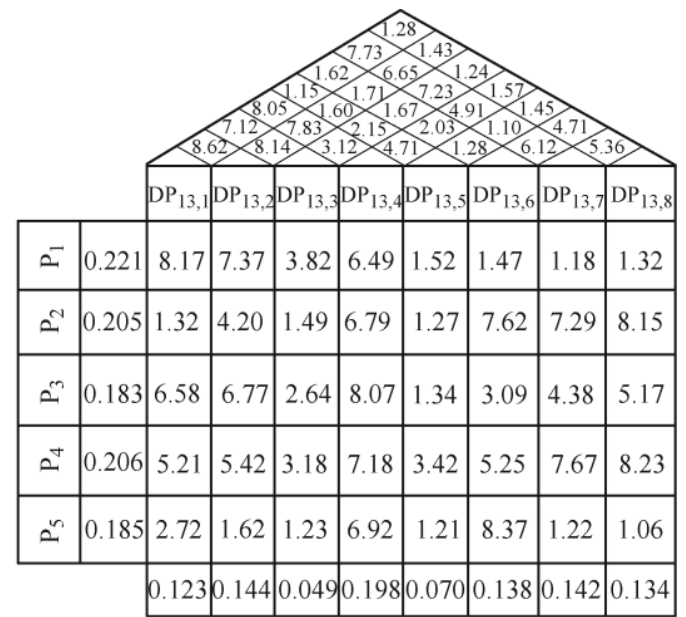

(b) 性能参数-设计参数关联关系矩阵

图 9 两级关联关系矩阵计算结果

\section{2 对比分析}

(1) 单隐层前馈神经网络训练算法对比。

为了验证极限学习机算法在求解单隐层前馈神 经网络时的优势, 对比分析了传统 BP 神经网络与 极限学习机 ELM 的训练速度和准确度, 结果如表 5 所示。可以看出, 基于 ELM 的工况识别模型将训 练时间大大缩短, 在训练速度上拥有极大的优势。 同时, 均方根误差(Root mean squared error, RMSE) 也低于 BP 模型, 拥有良好的准确度。ELM 模型在 保证训练精度的前提下, 极大地提高了神经网络的 训练效率。

表 5 神经网络求解算法性能对比

\begin{tabular}{ccc}
\hline 模型 & 训练时间 $/ \mathrm{s}$ & RMSE \\
\hline BP 神经网络 & 5.6958 & 0.2817 \\
极限学习机 & 0.0731 & 0.1935 \\
\hline
\end{tabular}

（2）性能退化评估方法对比。

为了验证多工况性能退化评估方法的可行性, 仍以 F13 变幅运动功能模块性能监测数据为基础, 在不考虑工况划分的前提下, 训练基准 GMM 模型, 然后按照 GMM 模型训练更新方法进行模型更新, 保持模型运行环境不变, 并选取相同监测时刻点的 GMM 星云图进行对比, 得到的混合高斯模型的高 斯单元状态空间分布如图 10a 所示, 图 10b 为副臂 工况下对应监测时刻的 GMM 模型。考虑工况识别 的高斯混合模型的数据状态空间分布与未考虑工况 进行性能退化评估的相比, 两者仅在初始工况下保 持 GMM 模型一致, 而在工况发生变动后, GMM 模型的高斯单元状态空间分布差别较大。事实上, 考虑工况变化的性能退化评估更符合工程实际。
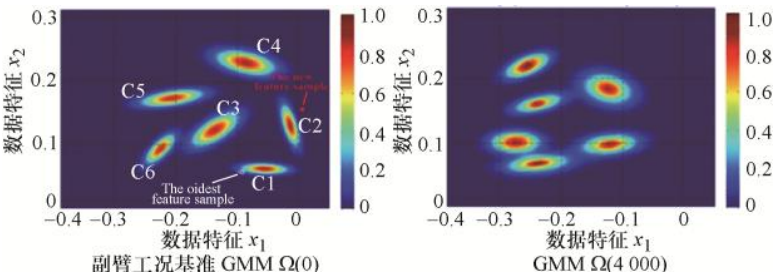

(a) 基于工况识别的性能评估模型变动


(b) 未考虑工况变化的性能评估模型变动

图 $10 \mathrm{GMM}$ 性能评估模型变动对比分析

(3) 关键功能模块识别方法对比。

为了验证性能数据驱动的关键功能模块识别方法 的有效性, 将所提方法与文献[18]中提出的顾客需求数 据驱动的功能重要度排序方法, 文献[19]中提出的基于 设计故障模式影响分析(Design failure mode effect analysis, DFMEA)的功能模块识别方法和文献[20]提 出的功能故障设计(Function failure design method, FFDM)分析矩阵的方法进行对比。表 6 展示了分别使 用上述四种方法识别出的关键功能模块。

表 6 采用不同方法得到的关键功能模块

\begin{tabular}{ccc}
\hline 决策依据 & 方法 & 关键功能模块 \\
\hline 顾客需求数据 & QFD & F11, F13, F21, F22, F33 \\
功能故障数据 & DFMEA & F12, F13, F14, F21, F31 \\
功能故障数据 & FFDM & F11, F13, F14, F21, F31 \\
性能监测数据 & 性能退化分析 & F12, F13, F21, F32, F34 \\
\hline
\end{tabular}


对比识别结果可以看出, 采用文献[19]和文 献[20]的方法识别出的关键功能模块基本一致, 而 使用基于性能退化分析的方法得到的关键功能模块 和传统方法有较大差异。采用设计 DFMEA 和 FFDM 矩阵的分析方法主要考虑了各功能模块的功 能故障和设计师的主观经验知识, 采用顾客需求数 据和 QFD 方法得到的关键功能模块与这两种方法 差异较大, 主要在于这类方法主要关注用户需求, 通过将用户需求映射到功能需求上确定关键的功能 模块。而本文所提出的方法采用产品运行过程中的 性能数据作为判断依据, 其分析过程相比上述方法 更为客观。得到退化严重的关键功能模块后, 可以 进一步识别出关键性能监测参数和关键设计参数。 这些信息可以为设计工程师在开发改进型产品的过 程中提供指导, 通过重新设计关键设计参数、替换 或修改关键功能模块、对已有产品的功能模块进行 改型借用等措施, 提高产品改型设计的效率, 保证 产品的运行服役性能稳定可靠。

\section{5 结论}

(1) 提出了基于极限学习机算法的运行工况识 别方法, 针对不同工况建立不同的性能退化评估模 型, 消除了工况变化对性能退化评估准确性的影响。

(2) 采用核主成分分析和高斯混合模型评估功 能模块的性能退化情况, 提出了基于滑动时间窗的 高斯混合模型更新策略, 提高了模型训练的效率; 在多工况下的性能退化评估的基础上, 识别出了关 键功能模块。

(3) 提出了基于性能监测数据聚类分析的关键 监测参数识别方法, 构建了 “性能监测参数一性能 参数一设计参数” 两级关联关系矩阵, 实现了从关 键监测参数到关键设计参数的映射, 为产品改进设 计提供了基础。

\section{参 考 文 献}

[1] SHIN J H, KIRITSIS D, XIROUCHAKIS P. Design modification supporting method based on product usage data in closed-loop PLM[J]. International Journal of Computer Integrated Manufacturing, 2015, 28(6) : 551-568.

[2] JANZ D, SIHN W, WARNECKE H J. Product redesign using value-oriented life cycle costing[J]. CIRP Annals Manufacturing Technology, 2005, 54(1): 9-12.

[3] 孟祥慧, 谢友柏, 戴旭东. 面向复杂产品时变性能设计
的理论与方法[J]. 机械工程学报, 2010, 46(1): 128-133. MENG Xianghui , XIE Youbai , DAI Xudong. Methodology of designing for time-varying performance of complex products[J]. Chinese Journal of Mechanical Engineering, 2010, 46(1): 128-133.

[4] ZASLAVSKY A, PERERA C, GEORGAKOPOULOS D. Sensing as a service and big data [C]. International Conference on Advances in Cloud Computing (ACC-2012), Bangalore, India, 2012: 21-29.

[5] ALANEZI K, MISHRA S. Design, implementation and evaluation of a smartphone position discovery service for accurate context sensing[J]. Computers \& Electrical Engineering, 2015, 44(C): 307-323.

[6] ASELMAA A, HERK M V, LAPRIE A, et al. Using a contextualized sensemaking model for interaction design: A case study of tumor contouring[J]. Journal of Biomedical Informatics, 2017, 65(C): 145-158.

[7] GHOSH D, OLEWNIK A, LEWIS K, et al. Cyber-empathic design: A data-driven framework for product design[J]. Journal of Mechanical Design, 2017, 139(9): 091401.

[8] NGUYEN V. Are you ready for me now? Mobile communication design for emergency department team availability[C]//Proceeding of the 34th ACM International Conference on the Design of Communication, 2016, $\quad 57$ : 1.

[9] 杨培林, 徐凯, 薛冲冲, 等. 基于模型检测的机电系统 FMEA 研究[J]. 机械工程学报, 2016, 52(16): 162-168. YANG Peilin, XU Kai, XUE Chongchong, et al. Study on FMEA for electromechanical systems based on model checking[J]. Journal of Mechanical Engineering, 2016, 52(16): 162-168.

[10] GARGAMA H, CHATURVEDI S K. Criticality assessment models for failure mode effects and criticality analysis using fuzzy logic[J]. IEEE Transactions on Reliability, 2011, 60(1): 102-110.

[11] ISERMANN R. Model-based fault-detection and diagnosis-status and applications[J]. Annual Reviews in Control, 2005, 29(1): 71-85.

[12] HUANG G B, ZHOU H, DING X, et al. Extreme learning machine for regression and multiclass classification[J]. IEEE Transactions on Systems Man \& Cybernetics Part B, 2012, 42(2): 513-529.

[13] CAO L J, CHUA K S, CHONG W K, et al. A comparison of PCA, KPCA and ICA for dimensionality reduction in 
support vector machine[J]. Neurocomputing, 2003, 55(1): 321-336.

[14] GAO C, ZHU Y, SHEN X, et al. Estimation of multiple networks in Gaussian mixture models[J]. Electronic Journal of Statistics, 2016， 10(1): 1133-1154.

[15] YANG M S, LAI C Y, LIN C Y. A robust EM clustering algorithm for Gaussian mixture models[J]. Pattern Recognition, 2012, 45(11): 3950-3961.

[16] ZENG J, KRUGER U, GELUK J, et al. Detecting abnormal situations using the Kullback-Leibler divergence[J]. Automatica, 2014, 50(11): 2777-2786.

[17] MODHAW D S, SPANGLER W S. Feature weighting in k-means clustering $[\mathrm{J}]$. Machine Learning, 2003, 52(3): 217-237.

[18] SMITH S, SMITH G, SHEN Y T. Redesign for product
innovation[J]. Design Studies, 2012, 33(2): 160-184.

[19] CHEN L H, KO W C. Fuzzy linear programming models for new product design using QFD with FMEA[J]. Applied Mathematical Model, 2009, 33(2): 633-647.

[20] STONE R B, TUMER I Y, VAN WIE M. The function-failure design method[J]. ASME Journal of Mechanical Design, 2005, 127(3): 397-407.

作者简介: 褚学宁(通信作者), 男, 1961 年出生, 教授, 博士研究生导 师。主要从事产品工程、服务科学与系统、工业信息工程等方面的科研 教学工作。

E-mail: xnchu@sjtu.edu.cn

陈汉斯, 男, 1991 年出生, 博士研究生。主要从事大型机械产品环境适 应性设计研究。

E-mail: scirocco@sjtu.edu.cn

马红占, 男, 1991 年出生, 博士研究生。主要从事数据驱动的产品改进 设计研究。

E-mail: mahongzhan@sjtu.edu.cn 